\title{
Effects of Kainic Acid and Radiofrequency Lesions of the Pulvinar on Visual Discrimination in the Monkey
}

\author{
SANDRA NAGEL-LEIBY ${ }^{1}$, D. B. BENDER ${ }^{2}$ and CHARLES M. BUTTER ${ }^{1}$ \\ ${ }^{\prime}$ Neuroscience Laboratory and Psychology Department, The University of Michigan, Ann Arbor, MI 48109 and ${ }^{2}$ Division of \\ Neurobiology, Physiology Department, State University of New York, Buffalo, NY 14226 (U.S.A.)
}

(Accepted September 27th, 1983)

Key words: monkeys - pulvinar — superior colliculus — visual discrimination — kainic acid — stimulus-response separation

\begin{abstract}
Monkeys with thermocoagulation or kainic acid lesions of the pulvinar and unoperated control monkeys were tested in two tasks: pattern discrimination retention and color discrimination learning in which the stimuli were located at the response sites or were separated spatially from them (S-R separation). The monkeys with kainic acid pulvinar lesions were mildly impaired in retention of the pattern discrimination, but were unimpaired in the color discrimination tasks with or without the S-R separation. The monkeys with thermocoagulation lesions, like monkeys with superior colliculus lesions in a prior study, were severely impaired in performing one of the color discrimination tasks with S-R separation. These findings suggest that: (a) the inferior pulvinar, unlike the superior colliculus, does not contribute to the performance of discriminations involving S-R separation; and (b) corticotectal projections traversing the pulvinar and destroyed by the thermocoagulation lesions are crucial to the performance of discriminations involving S-R separation. The results of an earlier experiment also suggested that interruption of corticotectal fibers passing through the pulvinar impairs performance in another task sensitive to superior colliculus lesions - spatial localization of light flashes. Thus, corticotectal projections may be crucial for the contribution of the colliculus to performance in a variety of visual tasks.
\end{abstract}

\section{INTRODUCTION}

Anatomical and physiological evidence strongly suggests that the primate pulvinar is involved in vision. The pulvinar receives the major ascending outflow of the superficial layers of the superior colliculus $^{5,17.27}$, as well as projections from striate, prestriate and inferotemporal cortex $4,10,26,30.33$. It projects, in turn, to widespread areas of prestriate and inferotemporal cortex ${ }^{6.12}$. The pulvinar is retinotopically organized ${ }^{1}$ and its cells have receptive field properties similar to those of neurons in striate cortex $^{2}$. Neither anatomical nor electrophysiological evidence, however, has revealed the role of the pulvinar in vision.

There are two different views of how the pulvinar might contribute to visual function, each based upon the functions of structures to which the pulvinar is anatomically connected. According to one view ${ }^{13}$, which emphasizes the extensive reciprocal connec- tions between pulvinar and prestriate and inferotemporal cortex, the pulvinar might play a role in visual discrimination learning. This view has received little experimental support. Lesions restricted to the pulvinar do not impair performance in a variety of visual discrimination problems ${ }^{13,25,31,32}$, despite the fact that performance of these same tasks is impaired by inferotemporal lesions ${ }^{15}$ and prestriate lesions ${ }^{19,25}$.

The second view ${ }^{29}$ of pulvinar function emphasizes the pulvinar as an anatomical link between the tectum and visual cortex, receiving input from the colliculus and projecting to prestriate cortex. On this view, one might expect pulvinar lesions to impair performance in tasks sensitive both to lesions of the colliculus and to lesions of prestriate cortex. Thus, the functions served by this pathway should be lost after a lesion at any stage along it. In a recent experimental test of this view, however, pulvinar lesions, unlike lesions of the superior colliculus ${ }^{9,18,23}$ or prestriate cortex ${ }^{19,20}$, did not impair localization of visual

Correspondence: D. B. Bender, Division of Neurobiology, Department of Physiology, SUNY/AB Medical School, 4234 Ridge Lea Road, Amherst, NY 14226, U.S.A. 
stimuli unless the pulvinar lesions also interrupted corticotectal fibers ${ }^{21}$.

One goal of the experiments reported here was to provide a further test of the above views of pulvinar function. We evaluated visual discrimination performance following pulvinar lesions in monkeys by testing postoperative retention of a preoperatively learned pattern discrimination, and postoperative acquisition of two color discriminations. Prestriate and inferotemporal cortex lesions each impair performance on these problems, whereas colliculus lesions do not ${ }^{7.16}$. Thus, if the first view of pulvinar function is correct, pulvinar lesions should also impair performance on these tasks. To test the second view, we examined the effect of pulvinar lesions on the performance of color discriminations in which the stimuli were spatially separated from the response sites. Both colliculus and prestriate lesions impair performance in this task 7.8 . If the second view is correct, pulvinar lesions should also impair this form of discrimination behavior.

Another goal of the present study was to test a suggestion arising from our findings that the impairment in localization following colliculus lesions could also be produced by radiofrequency pulvinar lesions, provided those lesions interrupted corticotectal fibers ${ }^{21}$. This observation suggested that the ability to localize visual stimuli is dependent on cortical projections to the colliculus. However, we could not rule out the possibility that destruction of both the pulvinar itself and corticotectal fibers as well was necessary to impair localization. In the present experiment, we compare the effects of two different kinds of pulvinar lesions on another task, in which, as mentioned previously, monkeys with colliculus lesions are impaired: color discrimination learning with a stimulus-response separation. In some monkeys, pulvinar lesions were made by thermocoagulation, which destroys both pulvinar cells and a substantial proportion of corticotectal fibers ${ }^{3}$. In other monkeys, pulvinar lesions were made by kainic acid injection, thus destroying pulvinar cells but leaving intact most corticotectal fibers ${ }^{3}$. A behavioral deficit common to the two lesion groups would indicate the contribution of the pulvinar per se; a deficit seen only following thermocoagulation lesions should indicate the contribution of the corticotectal fibers.

\section{METHODS}

\section{Subjects}

The subjects were 12 experimentally naive cynomolgous monkeys (Macaca fascicularis), 9 males and 3 females, ranging in weight from 3.2 to $9.7 \mathrm{~kg}$. They were individually housed and fed Purina monkey chow supplemented with fresh fruit. Isoniazid $(25 \mathrm{mg}$ ) was administered daily in a tablet to each monkey, as a prophylaxis against tuberculosis.

\section{Apparatus and behavioral procedures}

Training and testing were conducted in a Wisconsin General Testing Apparatus. When an opaque screen was raised, the monkey could displace plaques located over two recessed food wells, $33 \mathrm{~cm}$ apart, in a stimulus tray. Another screen, containing a oneway window through which the experimenter observed the monkey, separated the compartment with the stimulus tray from the experimenter.

After the monkeys learned to displace posterboard plaques from the foodwells in order to obtain small pieces of fruit, they were trained to perform a twochoice pattern discrimination task (plus vs square), which has been described in detail previously ${ }^{7}$. Within each testing session of 30 trials, the spatial positions of the two patterns was determined by a Gellermann series ${ }^{14}$. Training was terminated when the monkey attained 90 correct responses in 100 consecutive trials.

The monkeys were then assigned to one of 3 groups: a radiofrequency pulvinar lesion group $(\mathrm{n}=$ $5)$, a kainic acid pulvinar lesion group $(n=3)$ and an unoperated control group $(n=4)$. Approximately 2 weeks following surgery, or after a comparable waiting period for the unoperated control monkeys, all the animals were tested for retention of the preoperatively acquired pattern discrimination.

The monkeys were next tested in two color discrimination tasks, each of which involved two stages. The procedures employed in these tasks have been reported in detail elsewhere? The $7.6 \mathrm{~cm}$-square plaques bearing the color cues were held in runners attached to the stimulus tray, so that the monkeys could displace them from the food wells only by pushing the edge nearest to them. The near edges of the plaques, then, were the response sites. In the first stage of each discrimination task, the color cues, pro- 
vided by strips of colored paper pasted to the gray plaques, were presented at the edge of the plaque closest to the monkey. Thus, the first stage of each discrimination task involved no stimulus-response (S-R) separation. After the monkeys performed 18 correct responses in 20 consecutive trials, they were tested in the second stage, in which the color strips were located at the edge of the plaque farthest away from the animal. Thus, in the second stage of each discrimination task, unlike the first, there was a 7.6$\mathrm{cm}$ S-R separation. The colors used in the first discrimination were blue (positive cue) and yellow; the colors used in the second discrimination were tan (positive cue) and orange. In each session of 30 trials the position of the positive cue was determined by a Gellermann ${ }^{14}$ series.

\section{Surgical procedures}

The radiofrequency pulvinar lesions were made bilaterally by passing sufficient radiofrequency current through the tip of an electrode to raise its temperature to $73-80^{\circ} \mathrm{C}$ for $1 \mathrm{~min}$. Lesions were made at 4 sites in each side of the thalamus. The sites were chosen by first making microelectrode penetrations into the lateral geniculate nucleus in order to locate its caudal pole on the basis of geniculate cell receptivefield positions. This allowed us to destroy most of the inferior pulvinar with only minimal damage to the geniculate. A complete description of the recording procedures and other surgical procedures may be found elsewhere ${ }^{1}$. Kainic acid $(1 \mathrm{mg} / \mathrm{ml}$ in saline, $\mathrm{pH}$ adjusted to 7.2 ) was injected in $1.0-1.2 \mu \mathrm{l}$ volumes at 4 sites on each side of the thalamus through a $0.47 \mathrm{~mm}$ diameter cannula attached to a $5 \mu 1$ Hamilton syringe. Each injection lasted $4 \mathrm{~min}$. The surgical and recording procedures used to guide the cannula were the same as those used to make the radiofrequency lesions.

\section{Histological procedures}

Following the completion of testing, the monkeys were deeply anesthetized with ketamine hydrochloride and perfused intracardially with $0.9 \%$ saline followed by $10 \%$ formalin. The tissue containing the pulvinar was blocked in stereotaxic planes, further hardened in sucrose-formalin and embedded in albumin-gel. Frozen cross-sections $40 \mu \mathrm{m}$ in thickness were then cut. Every fifth section through the pulvi- nar was stained with thionin; every sixth section was stained by the Weil or Heidenhain-Woelcke method. The lesions were then plotted on standard drawings of the brain.

\section{RESULTS}

\section{Histological findings}

The electrolytic lesions all damaged the inferior pulvinar bilaterally (see Fig. 1). The extent of the inferior pulvinar lesions was assessed by first estimating the amount of damage to the inferior pulvinar in tracings of each cross-section and then averaging the estimates, each weighted by the size of the inferior pulvinar region, over all cross-sections. The degree of damage to other regions of the pulvinar and to other structures was estimated by direct inspection. Estimates of damage by radiofrequency lesions to the inferior pulvinar varied from 33\% (PE-4) to 89\% (PE5 ). In addition, the radiofrequency lesions involved varying degrees of damage to other regions of the pulvinar: all the monkeys with these lesions showed slight damage to the hippocampus and fornix. PE-1, PE-2 and PE-3 had damage to the caudal pole of the lateral geniculate nucleus. PE-3 had slight bilateral damage to the reticular nucleus of the thalamus and the stria terminalis and slight unilateral damage to the tail of the caudate nucleus (see Fig. 1).

The monkeys with kainic acid lesions, like those with radiofrequency lesions, all sustained bilateral damage to the inferior pulvinar (see Fig. 1); estimated by the same methods used with animals in the PE group, inferior pulvinar damage varied from $43 \%$ (PK-3) to $85 \%$ (PK-1). The kainic acid lesions also involved variable damage to other regions of the pulvinar, as well as bilateral damage to the caudal pole of the lateral geniculate nucleus. PK-1 and PK-2 had hippocampal damage; PK-1 also sustained damage to the reticular nucleus of the thalamus.

\section{Behavioral findings}

Table I shows the number of trials required to achieve criterion in each of the 3 tasks. Fig. 2 shows the savings scores in pattern discrimination retention and in the S-R separation stage of each color discrimination. Included in Table I and Fig. 2 are the scores of two groups of rhesus monkeys, one with superior colliculus lesions and a control group with subcortical 


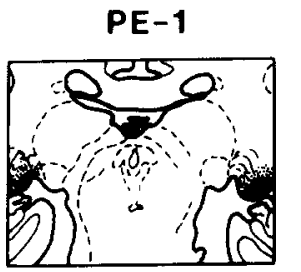

5.5

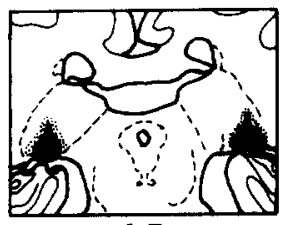

4.5

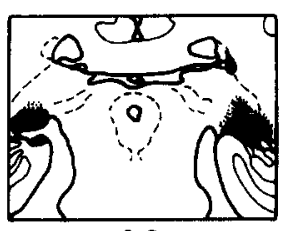

4.0

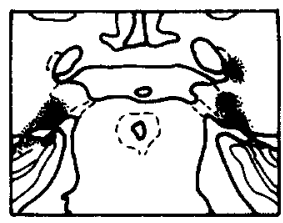

3.5

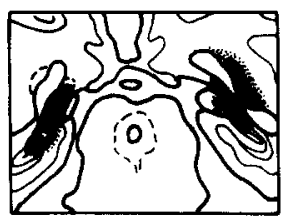

A

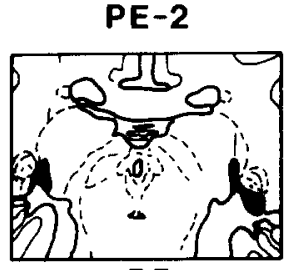

5.5
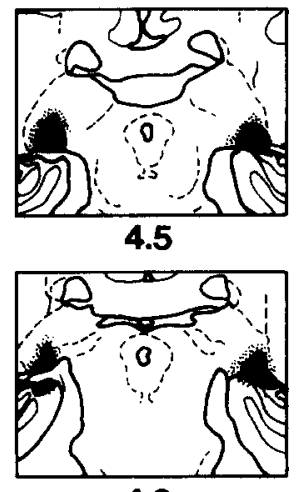

4.0

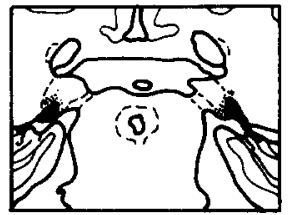

3.5

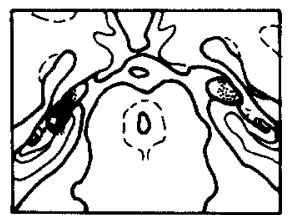

2.5

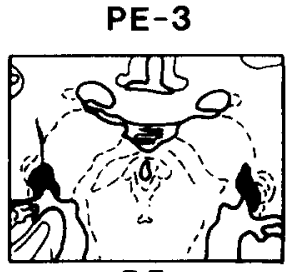

5.5

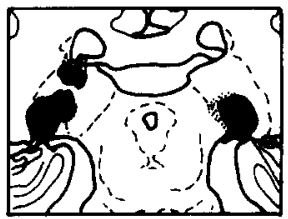

4.5

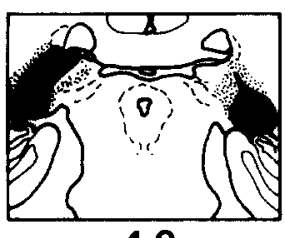

4.0

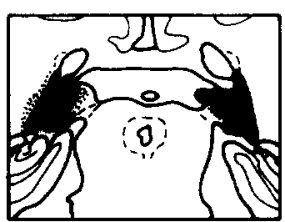

3.5

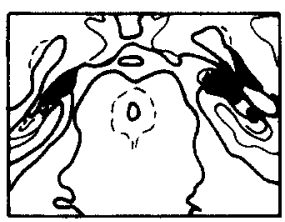

2.5
PE-4

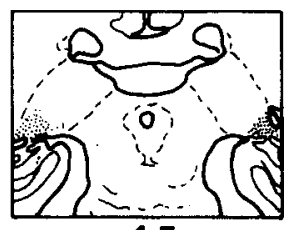

4.5

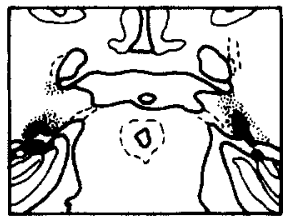

3.5

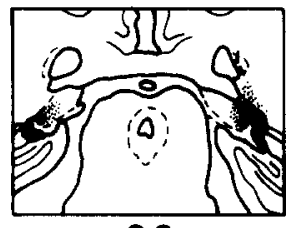

3.0

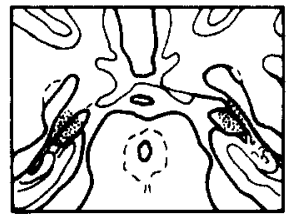

2.5

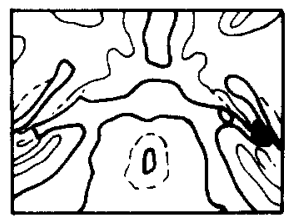

2.0
PE-5

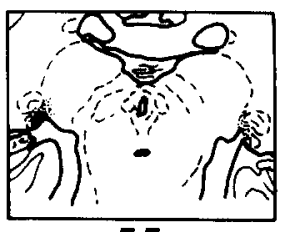

5.5

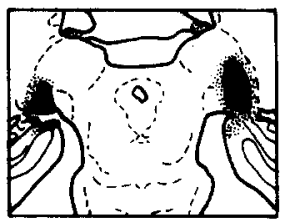

4.5

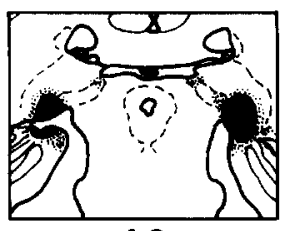

4.0

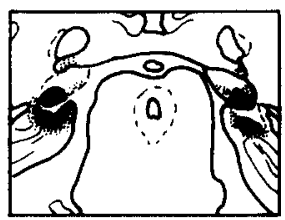

3.0

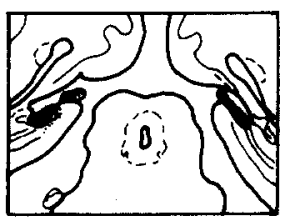

2.0

Fig. 1. A: cross-sections through the pulvinar in monkeys with radiofrequency pulvinar lesions (Group PE). Regions of total cell loss in black; regions of partial cell loss and gliosis in stippling.

lesions involving neither colliculus nor pulvinar. These animals were tested by the same procedures in the same apparatus?. In the statistical analyses reported below, the effects of pulvinar lesions were evaluated by one-tailed, Mann-Whitney U tests; $z$ scores were used when scores of monkeys in different groups were tied ${ }^{28}$. Since rhesus monkeys learn visual discriminations somewhat faster than do cynomolgous monkeys ${ }^{22}$, a finding confirmed by comparing the two species in preoperative acquisition of the pattern discrimination (see Table I), the scores of the subcortical control and of the colliculus lesion groups were not included in the statistical analyses.

Pattern discrimination. Neither pulvinar-lesioned group differed statistically from the unoperated control group in preoperative acquisition of the pattern discrimination. However, both pulvinar-lesioned groups showed somewhat poorer postoperative retention than did the unoperated control group. As shown in Table I and Fig. 2, the monkeys with radiofrequency lesions (Group PE) required slightly more trials to relearn the pattern discrimination and showed slightly poorer savings than the unoperated control monkeys $(z=1.75, P<0.05)$. Likewise, the monkeys with kainic acid lesions (Group PK) took more trials to relearn the pattern discrimination and showed poorer savings than the unoperated control monkeys ( $\mathrm{U}=0, P<0.03$ ). Group PE did not differ 


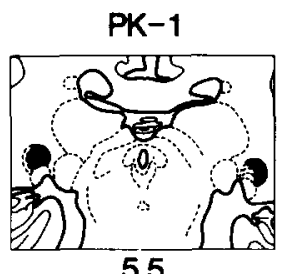

5.5
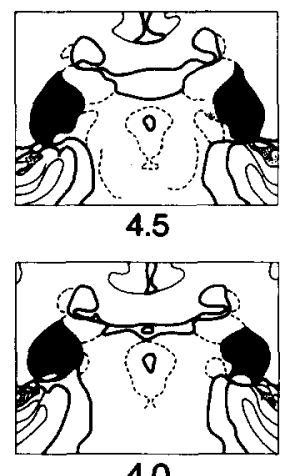

4.0
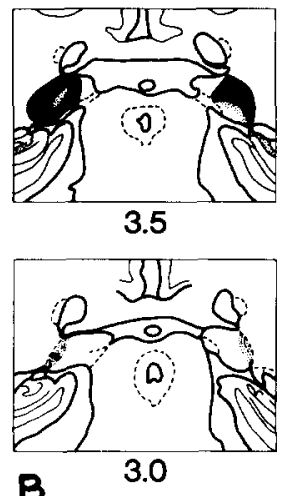

PK-2

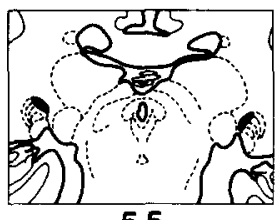

5.5
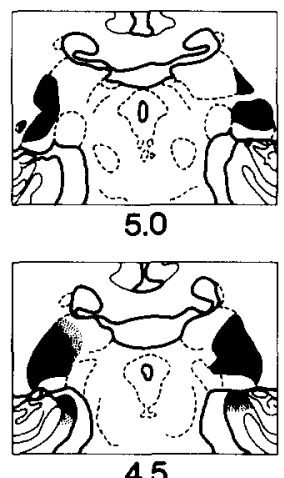

4.5

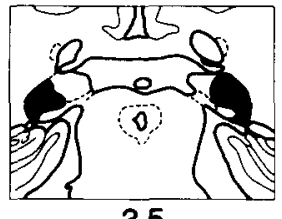

3.5

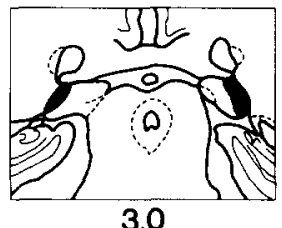

PK-3

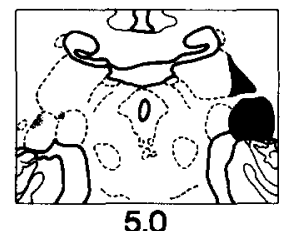

5.0
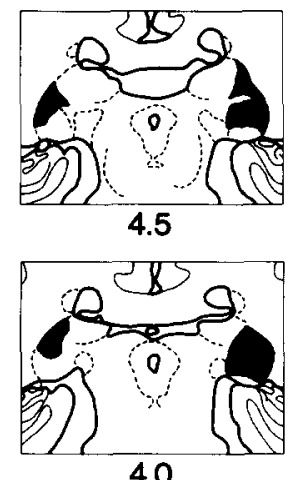

4.0

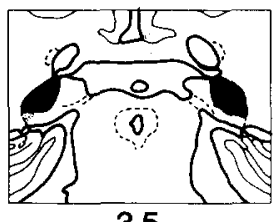

3.5

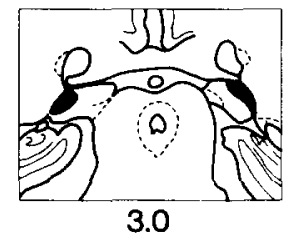

Fig. 1. B: cross-sections through the pulvinar in monkeys with kainic acid lesions (Group PK). Regions of total cell loss in black; regions of partial cell loss and gliosis in stippling.

on either measure from Group PK.

Color discriminations without $S$ - $R$ separation. Both pulvinar-lesioned groups required more trials to meet the criterion in the first stage of the first color discrimination task (blue vs yellow) than did the unoperated control group. However, only the difference between Group PE and controls reached significance ( $\mathrm{U}=2, P<0.04)$. In the second color discrimination task (tan vs orange), both pulvinar-lesioned groups again required more trials to learn than did the unoperated control group. However, none of the differences between the groups reached significance.

Color discriminations with $S$ - $R$ separation. Group PE took more trials to achieve criterion in the $S-R$ separation stage of the first color discrimination than either the unoperated control group or Group PK; however, no statistically significant group differences were found in this stage of testing, whether performance was measured by trials to relearn or percent savings. Nor were there any significant group differences when performance on the first 20 trials of relearning was examined, as might be expected if the pulvinar groups had shown less transfer from the stage with no $S-R$ separation to the $S-R$ separation stage. Group PE's poor performance failed to result in significant group differences in part because of the large variability of all animals' scores on this task, and in part because of the poor performance of the control animals. The latter is particularly evident when the scores of the subcortical control and collicu- 
TABLE I

Trials to criterion in pattern discrimination learning and retention, and in color discriminations with and without $S$ - $R$ separation

\begin{tabular}{|c|c|c|c|c|c|c|}
\hline \multirow[t]{2}{*}{ Monkey } & \multicolumn{2}{|c|}{$\begin{array}{l}\text { Pattern } \\
\text { discrimination }\end{array}$} & \multicolumn{2}{|l|}{$\begin{array}{l}\text { Blue-Yellow } \\
\text { discrimination }\end{array}$} & \multicolumn{2}{|l|}{$\begin{array}{l}\text { Tan-Orange } \\
\text { discrimination }\end{array}$} \\
\hline & $\begin{array}{l}\text { Pre-op. } \\
\text { learn. }\end{array}$ & $\begin{array}{l}\text { Post-op. } \\
\text { retent. }\end{array}$ & $\begin{array}{l}\text { Stage } 1 \text {, } \\
\text { No } S-R \text { separat. }\end{array}$ & $\begin{array}{l}\text { Stage 2, } \\
\text { S-R separat. }\end{array}$ & $\begin{array}{l}\text { Stage } 1 \text {, } \\
\text { No S-R separat. }\end{array}$ & $\begin{array}{l}\text { Stage 2, } \\
\text { S-R separat. }\end{array}$ \\
\hline \multicolumn{7}{|c|}{ Unoperated control } \\
\hline 1 & 279 & 0 & 30 & 30 & 43 & 35 \\
\hline 2 & 290 & 0 & 82 & 221 & 120 & 98 \\
\hline 3 & 366 & 5 & 142 & 0 & 283 & 58 \\
\hline 4 & 215 & 7 & 55 & 80 & 150 & 120 \\
\hline$\overline{\mathrm{x}}$ & 287 & 3 & 77 & 83 & 149 & 78 \\
\hline \multicolumn{7}{|c|}{ Group PE } \\
\hline 1 & 331 & 363 & 118 & 67 & 9 & 367 \\
\hline 2 & 440 & 17 & 95 & 431 & 160 & 450 \\
\hline 3 & 260 & 20 & 377 & 160 & 95 & $500^{*}$ \\
\hline 4 & 410 & 0 & 185 & $500^{*}$ & 742 & $1606^{*}$ \\
\hline 5 & 380 & 20 & 548 & 20 & 556 & $1036^{*}$ \\
\hline$\overline{\mathbf{x}}$ & 364 & 84 & 265 & 236 & 312 & 792 \\
\hline \multicolumn{7}{|c|}{ Group PK } \\
\hline 1 & 161 & 47 & 215 & 0 & 250 & 100 \\
\hline 2 & 472 & 114 & 118 & 5 & 444 & 53 \\
\hline 3 & 166 & 9 & 141 & 432 & 248 & 205 \\
\hline$\overline{\mathrm{x}}$ & 208 & 57 & 158 & 146 & 314 & 119 \\
\hline \multicolumn{7}{|c|}{ Sub-cort. control $\$$} \\
\hline 1 & 171 & 0 & 53 & 0 & 7 & 0 \\
\hline 2 & 156 & 0 & 48 & 36 & 50 & 0 \\
\hline 3 & 163 & 0 & 25 & 33 & 57 & 41 \\
\hline 4 & 101 & 0 & 91 & 8 & 77 & 22 \\
\hline$\overline{\mathbf{x}}$ & 148 & 0 & 54 & 19 & 48 & 16 \\
\hline \multicolumn{7}{|c|}{ Sup. coll. ${ }^{\S}$} \\
\hline 1 & 110 & 5 & 7 & 155 & 25 & 180 \\
\hline 2 & 200 & 5 & 64 & 51 & 26 & 45 \\
\hline 3 & 140 & 5 & 40 & 79 & 65 & 97 \\
\hline 4 & 170 & 5 & 56 & 91 & 84 & 33 \\
\hline 5 & 220 & 5 & 21 & 29 & 94 & 119 \\
\hline 6 & 300 & 5 & 34 & 66 & 49 & 54 \\
\hline$\overline{\mathbf{x}}$ & 190 & 5 & 37 & 78 & 57 & 88 \\
\hline
\end{tabular}

* Training was stopped when the errors in Stage 2 were 4 times the errors in Stage 1.

\& Data from ref. 7.

lus-lesioned animals in our previous experiment are compared with those of the control and pulvinar-lesioned animals in the present study (see Table I and Fig. 2).

Clear group differences were found in the S-R separation stage of the second color discrimination task. Group PE showed a severe impairment similar to that shown by colliculus-lesioned animals: all the Group PE animals required more trials to reattain criterion than any control animal, and all showed negative savings ( $U=0, P<0.01)$. Furthermore, the Group PE monkeys made significantly more errors during the first 20 trials of relearning than did the controls $(U=0, P<0.02)$. In contrast, the kainic 

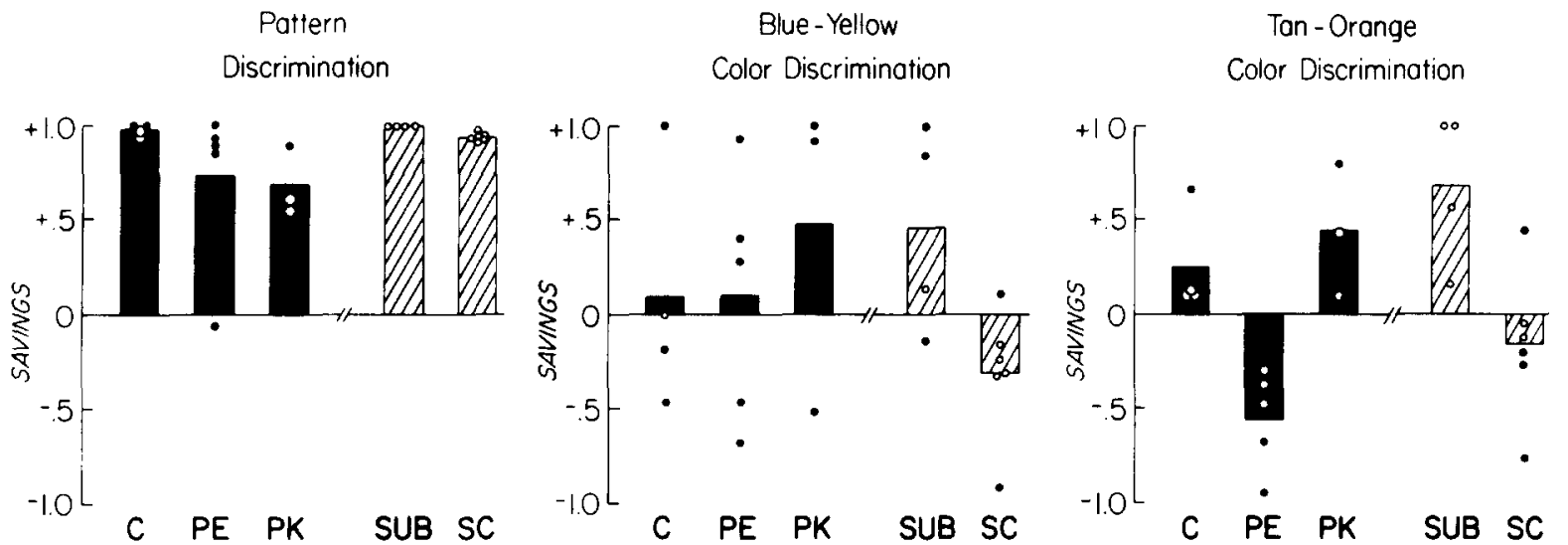

Fig. 2. Mean (bars) and individual (circles) savings scores for unoperated control monkeys (C), monkeys with radiofrequency pulvinar lesions (PE), and monkeys with kainic acid pulvinar lesions (PK). The savings scores of animals with subcortical lesions (SUB) and superior colliculus lesions (SC) are taken from a previous study (ref. 7). Savings scores are calculated from the formula $(x-y) /(x+y)$. For the pattern discrimination, $x$ and $y$ are the pre- and postoperative trials to criterion, respectively; for the color discriminations, $x$ and $y$ are trials to criterion for stage 1 (no S-R separation) and stage 2 (S-R separation), respectively.

acid-lesioned animals did not differ from controls, either in trials to reattain the criterion or in savings scores. Nor did they show impaired transfer as measured by performance on the first 20 trials of relearning. Furthermore, Group PK took fewer trials to relearn and showed better savings in relearning than did Group PE ( $\mathrm{U}=0, P<0.02)$.

Finally, there was no relationship between the degree of damage to the tectorecipient or other regions of the pulvinar and the severity of the behavioral impairments. Damage to other structures was also unrelated to the behavioral impairments.

\section{DISCUSSION}

The findings of the present experiment are relevant to two views of pulvinar function, both of which are based on this structure's anatomical connections. One of these views holds that the pulvinar shares functions with both the superior colliculus and prestriate cortex, between which it provides an anatomical link. According to this view, one might expect that kainic acid lesions of the inferior pulvinar would impair performance in a color discrimination task with $S-R$ separation, a task that is sensitive to prestriate $^{7}$ and collicular ${ }^{7,8}$ lesions. The finding that monkeys with kainic acid lesions that destroyed a large part of the inferior pulvinar bilaterally were not impaired in this task is not consistent with this view. Further evidence against this view is provided by our finding that electrolytic pulvinar lesions fail to impair performance in another task sensitive to collicular ${ }^{9}$ and prestriate lesions ${ }^{19,20}$, localization of visual stimuli, unless the lesions interrupted corticotectal fibers $^{21}$. Together, these findings imply that the tectothalamic-cortical pathway does not contribute to functions involved in either the S-R separation or localization task.

The second view of pulvinar function suggests that the pulvinar shares functions with extrastriate visual areas (prestriate and inferotemporal cortex) with which it has reciprocal connections. If this view is correct, one might expect that kainic acid lesions of the inferior and lateral pulvinar would impair performance in visual discrimination tasks that are sensitive to lesions of these extrastriate visual cortical areas. The present findings provide partial support for this view: kainic acid lesions of large portions of the inferior and lateral pulvinar led to mild deficits in pattern discrimination retention. Furthermore, these deficits were not likely to be due to damage to the caudal pole of the lateral geniculate nucleus, for there was no relationship between the extent of damage to this structure and the severity of impairments in pattern discrimination relearning in either pulvinar lesioned group. It should be noted that deficits in pattern discrimination retention, even mild ones, have not been found in monkeys with histologically verified lesions restricted to the pulvinar ${ }^{13,25}$. However, these studies used rhesus monkeys, which learn visual discrimina- 
tions more rapidly than do cynomolgous monkeys ${ }^{22}$, the species used in the present study. In fact, the cynomolgous monkeys in this study required approximately twice as many trials to learn the plus vs square discrimination preoperatively compared to rhesus monkeys that learned the same discrimination by the same procedures (see Table I). Thus, it is possible that pulvinar lesions impair performance in difficult discrimination tasks but not in easy ones. This possibility might account for the kainic acid-lesioned monkeys' lack of impairment in initial learning of the relatively easy color discrimination tasks as well as the severe impairment of pulvinar-lesioned monkeys in learning a very difficult pattern discrimination ${ }^{11}$.

The present findings provide support for the conclusion that cortical projections to the superior colliculus play an important role in the performance of visual discriminations involving $S-R$ separation. Radiofrequency pulvinar lesions, but not kainic acid lesions of similar size and locus, resulted in severe impairments in the S-R separation stage of the second color discrimination. Moreover, these impairments were as severe as those found in monkeys with either colliculus or prestriate lesions 7 . Our previous finding that radiofrequency pulvinar lesions result in locali-

\section{REFERENCES}

1 Bender, D. B., Retinotopic organization of macaque pulvinar, J. Neurophysiol., 46 (1981) 672-693.

2 Bender, D. B., Receptive field properties of neurons in the macaque inferior pulvinar, J. Neurophysiol., 48 (1982) 1-17.

3 Bender, D. B. and Baizer, J. S., Anterograde degeneration in the superior colliculus following kainic acid and radiofrequency lesions of the macaque pulvinar, in preparation.

4 Benevento, L. A. and Davis, B., Topographical projections of the prestriate cortex to the pulvinar nuclei in the macaque monkey: an autoradiographic study, Exp. Brain Res., 30 (1977) 405-424.

5 Benevento, L. A. and Fallon, J., The ascending projections of the superior colliculus in the rhesus monkey, J. comp. Neurol., 160 (1975) 339-362.

6 Benevento, L. A. and Rezak, M., The cortical projections of the inferior pulvinar and adjacent lateral pulvinar in the rhesus monkey (Macaca mulatta): an autoradiographic study, Brain Research, 108 (1976) 1-24.

7 Butter, C. M., Effects of superior colliculus, striate and prestriate lesions on visual sampling in rhesus monkeys, $J$. comp. physiol. Psychol., 87 (1974) 905-917.

8 Butter, C. M., Contrasting effects of lateral striate and superior colliculus lesions on visual discrimination performance in thesus monkeys, J. comp. physiol. Psychol., 93 (1979) $522-537$ zation deficits only if they reduce the number of fibers in the brachium of the superior colliculus ${ }^{21}$, suggests that corticotectal projections may also play an important role in spatial localization. Thus, these projections may be crucial for performance not only in discriminations involving S-R separation, but in other visual tasks as well.

One factor considered crucial for performance in S-R separation tasks is the ability to move the eyes or shift attention from the response sites to the stimuli24. Likewise, the ability to shift attention toward peripheral targets is presumably important in spatial localization. Thus, it seems likely that corticotectal projections play a major role in moving the eyes and attention through space.

\section{ACKNOWLEDGEMENTS}

Supported by Research Grants MH26489 from the National Institute of Mental Health and EY02254 from the National Eye Institute. The authors thank Beverly Caffery for histological assistance and Judith Lehman, Marylin Hoy and Linda Smith for their editorial assistance in preparing the manuscript.

9 Butter, C. M., Weinstein, C., Bender, D. B. and Gross, C. G., Localization and detection of visual stimuli following superior colliculus lesions in rhesus monkeys, Brain Research, 156 (1978) 33-49.

10 Campos-Ortega, J. and Hayhow, W., On the organization of the visual cortical projection to the pulvinar in Macaca mulatta, Brain Behav. Evol., 6 (1972) 394-423.

11 Chalupa, L. M., Coyle, R. S. and Lindsay, D. B., Effect of pulvinar lesions on visual pattern discrimination in monkeys, J. Neurophysiol., 39 (1976) 345-369.

12 Chow, K.-L., A retrograde cell degeneration study of the cortical projection field of the pulvinar in the monkey, $J$. comp. Neurol., 93 (1950) 313-340.

13 Chow, K.-L., Lack of behavioral effects following destruction of some thalamic association nuclei in monkey, Arch. Neurol. Psychiat., 71 (1954) 762-771.

14 Gellermann, S., Chance order of alternating stimuli in visual discrimination experiments, J. genet. Psychol, 42 (1933) 207-208.

15 Gross, C. G., Visual functions of inferotemporal cortex. In R. Jung (Ed.), Handbook of Sensory Physiology, Vol. VII/3B, Springer, Berlin, 1973, pp. 451-482.

16 Gross, C. G., Cowey, A. and Manning, F. J., Further analysis of visual discrimination deficits following foveal prestriate and inferotemporal lesions in rhesus monkeys, $J$. comp. physiol. Psychol., 76 (1971) 1-7.

17 Harting, J. K., Huerta, M. F., Frankfurter, A. J., Stominger, N. L. and Royce, G. J.. Ascending pathways from the 
monkey superior colliculus: an autoradiographic analysis, J. comp. Neurol., 192 (1980) 853-882.

18 Keating, E. G., Impaired orientation after primate tectal lesions, Brain Research, 67 (1974) 538-541.

19 Keating, E. G., Effects of prestriate and striate lesions on the monkey's ability to locate and discriminate visual forms, Exp. Neurol., 47 (1975) 16-25.

20 Keating, E. G., Residual spatial vision in the monkey after removal of striate and preoccipital cortex, Brain Research, 187 (1980) 271-290.

21 Leiby, C. C., III, Bender, D. B. and Butter, C. M., Localization and detection of visual stimuli in monkeys with pulvinar lesions, Exp. Brain Res., 48 (1982) 449-454.

22 Leiby, C. C., Campbell, A. L. and Butter, C. M., Comparison of visual discrimination performance of rhesus and cynomolgous monkeys, Primate Newsletter, 17 (1978) 1-5.

23 MacKinnon, D. A., Gross, C. G. and Bender, D. B., A visual deficit after superior colliculus lesions in monkeys, Acta neurobiol. exp., 36 (1976) 169-180.

24 Meyer, D., Treichler, F. and Meyer, P., Discrete trial training techniques and stimulus variables. In A. Schrier, H. Harlow and F. Stollnitz (Eds.), Behavior of Non-Human Primates, Vol. 1, Academic Press, New York, 1965, pp. $1-49$.

25 Mishkin, M., Cortical visual areas and their interactions. In A. G. Karczmar and J. C. Eccles (Eds.), Brain and Human Behavior, Springer, New York, 1972, pp. 187-208.

26 Ogren, M. P. and Hendrickson, A. E., Pathways between striate cortex and subcortical regions in Macaca mulatta and Saimiri sciureus: Evidence for a reciprocal pulvinar connection, Exp. Neurol., 53 (1976) 780-800.

27 Partlow, G. D., Colonnier, M. and Szabo, J., Thalamic projections of the superior colliculus in the rhesus monkey, Macaca mulatta: a light and electron microscopic study, $J$. comp. Neurol., 171 (1977) 285-318.

28 Siegel, S., Nonparametric Statistics for the Behavioral Sciences, McGraw-Hill, New York, 1956.

29 Snyder, M. and Diamond, I. T., The organization and function of the visual cortex in the tree shrew, Brain Behav. Evol., 1 (1968) 244-288.

30 Ungerleider, L. G., Galkin, T. W. and Mishkin, M., Visuotopic organization of projections from striate cortex to inferior and lateral pulvinar in rhesus monkey, J. comp. Neurol., 217 (1983) 137-157.

31 Ungerleider, L. G., Ganz, L. and Pribram, K. H., Size constancy in rhesus monkeys: effects of pulvinar, prestriate and inferotemporal lesions, Exp. Brain Res., 27 (1977) 251-269.

32 Ungerleider, L. G. and Pribram, K. H., Inferotemporal versus combined pulvinar-prestriate lesions in the rhesus monkey: effects on color, object and pattern discrimination, Neuropsychologia, 15 (1977) 481-498.

33 Whitlock, P. G. and Nauta, W. J. H., Subcortical projections from the temporal neocortex in Macaca mulatta, $J$. comp. Neurol., 106 (1956) 183-212. 